PROCEEDINGS OF THE

AMERICAN MATHEMATICAL SOCIETY

Volume 138, Number 2, February 2010, Pages 495-504

S 0002-9939(09)10103-X

Article electronically published on September 11, 2009

\title{
A MATRIX SUBADDITIVITY INEQUALITY FOR SYMMETRIC NORMS
}

\author{
JEAN-CHRISTOPHE BOURIN \\ (Communicated by Marius Junge) \\ Dedicated to Françoise Lust-Piquard, with affection
}

\begin{abstract}
Let $f(t)$ be a non-negative concave function on $[0, \infty)$. We prove that

$$
\|f(|A+B|)\| \leq\|f(|A|)+f(|B|)\|
$$

for all normal $n$-by- $n$ matrices $A, B$ and all symmetric norms. This result has several applications. For instance, for a Hermitian $\mathbb{A}=\left[A_{i, j}\right]$ partitioned in blocks of the same size,

$$
\|f(|\mathbb{A}|)\| \leq\left\|\sum f\left(\left|A_{i, j}\right|\right)\right\|
$$
\end{abstract}

We also prove, in a similar way, that given $Z$ expansive and $A$ normal of the same size,

$$
\left\|f\left(\left|Z^{*} A Z\right|\right)\right\| \leq\left\|Z^{*} f(|A|) Z\right\| \text {. }
$$

\section{SOME RECENT RESUltS FOR POSITIVE OPERATORS}

Several nice inequalities for concave functions of operators have been recently established in a series of papers [5], 8, 7] and [6]. Most of these results are matrix versions of the obvious inequality

$$
f(a+b) \leq f(a)+f(b)
$$

for non-negative concave functions $f$ on $[0, \infty)$ and scalars $a, b \geq 0$. By matrix version we mean a suitable extension where scalars are replaced by $n$-by- $n$ matrices, i.e., operators on an $n$-dimensional Hilbert space $\mathcal{H}$. For instance, we have [8]:

Theorem 1.1. Let $A, B \geq 0$ and let $f:[0, \infty) \rightarrow[0, \infty)$ be concave. Then, for all symmetric norms,

$$
\|f(A+B)\| \leq\|f(A)+f(B)\| .
$$

As usual, capital letters $A, B, \ldots$ stand for operators, $A \geq 0$ refers to positive semi-definite, and a symmetric norm (or unitarily invariant) satisfies $\|A\|=\|U A V\|$ for all $A$ and all unitaries $U, V$. Thus, up to symmetric norms, the basic inequality (1) still holds on the cone of positive operators. This subadditivity result for norms cannot be extended to the determinant, even in the case of an operator concave

Received by the editors November 5, 2008, and, in revised form, June 8, 2009.

2000 Mathematics Subject Classification. Primary 15A60, 47A30, 47A60.

Key words and phrases. Matrix inequalities, symmetric norms, normal operators, concave functions.

(C)2009 American Mathematical Society Reverts to public domain 28 years from publication 
function such as $f(t)=\sqrt{t}$. The most elementary case in the above theorem is for the trace norm. Then, the result can be restated as a famous trace inequality [11]:

Rotfel'd inequality. Let $f$ be a concave function on $[0, \infty)$ such that $f(0) \geq 0$. Then, for all $A, B \geq 0$,

$$
\operatorname{Tr} f(A+B) \leq \operatorname{Tr}(f(A)+f(B)) .
$$

In the matrix setting, the concavity assumption is quite crucial as shown in the following simple remark 13 :

Let $f:[0, \infty) \rightarrow[0, \infty)$ be continuous with $f(0)=0$. If

$$
\operatorname{Tr} f(A+B) \leq \operatorname{Tr}(f(A)+f(B))
$$

holds for all two-by-two positive matrices $A, B$, then $f$ is concave.

To prove this statement, take for $s, t>0$,

$$
A=\frac{1}{2}\left(\begin{array}{cc}
s & \sqrt{s t} \\
\sqrt{s t} & t
\end{array}\right), \quad B=\frac{1}{2}\left(\begin{array}{cc}
s & -\sqrt{s t} \\
-\sqrt{s t} & t
\end{array}\right)
$$

and observe that the trace inequality means that $f$ is concave.

Theorem 1.1 closed a list of papers of several authors, including Ando-Zhan [1] and Kosem 10. However, it remained natural to ask whether this result could be extended to the set of all Hermitian, or even all normal, operators. We noticed a partial answer in [6]:

Theorem 1.2. Let $A, B \geq 0$ and let $f:[0, \infty) \rightarrow[0, \infty)$ be concave and e-convex. Then, for all symmetric norms,

$$
\|f(|A+B|)\| \leq\|f(|A|)+f(|B|)\| .
$$

Here the $e$-convexity property of $f$ means that $f\left(e^{t}\right)$ is convex on $(-\infty, \infty)$. In particular, the theorem holds for the power functions $f(t)=t^{p}, 1 \geq p \geq 0$. This result for normal operators entails several estimates for block matrices. A special case involving an operator partitioned into four normal blocks $A, B, C, D$ of the same size is

$$
\left\|\left|\left(\begin{array}{ll}
A & B \\
C & D
\end{array}\right)\right|^{p}\right\| \leq\left\||A|^{p}+|B|^{p}+|C|^{p}+|D|^{p}\right\|
$$

for all symmetric norms and $0 \leq p \leq 1$. These estimates, comparing an operator on $\mathcal{H} \oplus \mathcal{H}$ with a related operator on $\mathcal{H}$, differ from the usual ones in the literature where the norm of the full matrix is evaluated with the norms of its blocks, for instance, [9] and [3. In the subsequent sections we solve the conjectures in $[\underline{6}$ by showing that the assumption of e-convexity is not necessary in Theorem 1.2, in its application to block matrices and in some related inequalities. The proof of Theorem 1.2 given in [7] reduced to the positive case by using the fact that for any normal $A, B$ and any non-negative e-convex functions $f(t)$, we have

$$
\|f(|A+B|)\| \leq\|f(|A|+|B|)\|
$$

for all symmetric norms. This is no longer true if the e-convexity assumption is dropped. In fact, one can easily find two-by-two positive semi-definite matrices $A$, $B$ and a non-negative concave function $f(t)$ on $[0, \infty)$ such that

$$
\|f(|A-B|)\|>\|f(|A|+|B|)\|
$$


for all symmetric norms which are not a multiple scalar of the usual operator norm. For instance, take $f(t)=\min \{t, \sqrt{2} / 2\}$ and

$$
A=\left(\begin{array}{ll}
1 & 0 \\
0 & 0
\end{array}\right), \quad B=\left(\begin{array}{ll}
1 / 2 & 1 / 2 \\
1 / 2 & 1 / 2
\end{array}\right) .
$$

The main point of the forthcoming proof is to overcome this difficulty. This proof can be adapted in order to obtain a version for normal operators of the following companion result to Theorem 1.1:

Theorem 1.3. Let $A \geq 0$ and let $Z$ be expansive. If $f:[0, \infty) \rightarrow[0, \infty)$ is concave, then, for all symmetric norms,

$$
\left\|f\left(Z^{*} A Z\right)\right\| \leq\left\|Z^{*} f(A) Z\right\| .
$$

\section{SUbADDITIVITY RESUlTS FOR NORMAL OPERATORS}

We have the following norm inequalities:

Theorem 2.1. Let $A, B$ be normal and let $f:[0, \infty) \rightarrow[0, \infty)$ be concave. Then, for all symmetric norms,

$$
\|f(|A+B|)\| \leq\|f(|A|)+f(|B|)\| .
$$

Corollary 2.2. Let $Z=A+i B$ be a decomposition into real and imaginary parts, and let $f:[0, \infty) \rightarrow[0, \infty)$ be concave. Then, for all symmetric norms,

$$
\|f(|Z|)\| \leq\|f(|A|)+f(|B|)\| .
$$

This is a matrix version of the obvious inequality $f(|z|) \leq f(|a|)+f(|b|)$ for complex numbers $z=a+i b$. Since non-negative concave functions on $[0, \infty)$ are non-decreasing, we actually have $f(|z|) \leq f(|a|+|b|) \leq f(|a|)+f(|b|)$. But the left inequality cannot be extended to matrices. Indeed it is easy to find two-by-two matrices $Z=A+i B$ (a simple example is given with $A, B$ defined in (2)) with the eigenvalue relation

$$
\lambda_{2}(|A|+|B|)<\lambda_{2}(|Z|)<\lambda_{1}(|Z|)<\lambda_{1}(|A|+|B|) .
$$

Thus, there are non-negative concave functions such as $f(t)=\min \left\{t, \lambda_{2}(|Z|)\right\}$ such that

$$
\|f(|Z|)\|>\|f(|A|+|B|)\|
$$

for all symmetric norms which are not a multiple scalar of the usual operator norm.

Let $A, B$ be general operators. Applying Theorem 2.1 to the Hermitian operators

$$
\left(\begin{array}{cc}
0 & A^{*} \\
A & 0
\end{array}\right) \quad \text { and } \quad\left(\begin{array}{cc}
0 & B^{*} \\
B & 0
\end{array}\right)
$$

we obtain

$$
\left\|\left(\begin{array}{cc}
f(|A+B|) & 0 \\
0 & f\left(\left|A^{*}+B^{*}\right|\right)
\end{array}\right)\right\| \leq\left\|\left(\begin{array}{cc}
f(|A|)+f(|B|) & 0 \\
0 & f\left(\left|A^{*}\right|\right)+f\left(\left|B^{*}\right|\right)
\end{array}\right)\right\|
$$

so that letting $B=A^{*}$ yields:

Corollary 2.3. If $f:[0, \infty) \rightarrow[0, \infty)$ is concave, then, for all $Z$ and all symmetric norms,

$$
\left\|f\left(\left|Z+Z^{*}\right|\right)\right\| \leq\left\|f(|Z|)+f\left(\left|Z^{*}\right|\right)\right\| .
$$


Note that equality occurs in Corollary 2.3 whenever $f(0)=0$ and

$$
Z=\left(\begin{array}{cc}
0 & 0 \\
X & 0
\end{array}\right)
$$

where $X$ is arbitrary. Note also that it may happen that

$$
\left\|f\left(\left|Z+Z^{*}\right|\right)\right\|>\left\|f\left(|Z|+\left|Z^{*}\right|\right)\right\|
$$

for some concave functions and some symmetric norms, for instance when

$$
Z=\left(\begin{array}{lll}
0 & 1 & 0 \\
0 & 0 & 1 \\
0 & 0 & 0
\end{array}\right)
$$

and the norm is the sum of the two largest singular values.

At the end of this section, we will see some application of Theorem 2.1 to partitioned operators. Now, we turn to the proof of Theorem 2.1. We start by recalling the Ky Fan Principle. The Ky Fan $k$-norms of $A, k=1,2, \ldots, n$, are defined as the sum of its $k$ largest singular values,

$$
\|A\|_{(k)}=\sum_{j=1}^{k} \lambda_{j}(|A|) .
$$

Let $A, B$ be such that $\|A\|_{(k)} \leq\|B\|_{(k)}$ for all $k=1,2, \ldots, n$. Then, the vector of the singular values of $A$ lies in the convex hull of the permuted singular values of $B$ multiplied by \pm 1 ,

$$
\left(\lambda_{1}(|A|), \ldots, \lambda_{n}(|A|)\right) \in \operatorname{conv}_{\sigma}\left( \pm \lambda_{\sigma(1)}(|B|), \ldots, \pm \lambda_{\sigma(n)}(|B|)\right) .
$$

This can be proved by using the hyperplane separation process to reach a contradiction; see [12] for details and 2] for alternative proofs. From this convexity statement follows a useful fact:

Ky Fan principle. Suppose that $\|A\|_{(k)} \leq\|B\|_{(k)}$ for all Ky Fan $k$-norms. Then, we have $\|A\| \leq\|B\|$ for all symmetric norms.

We also need two elementary, well-known lemmas. For $A, B \geq 0$ it is sometimes convenient to write $A \prec_{w} B$ to mean that $\|A\| \leq\|B\|$ for all symmetric norms.

Lemma 1. Let $A, B, X, Y \geq 0$ such that $B \prec_{w} Y$ and $A \prec_{w} X$. Then,

$$
\left(\begin{array}{cc}
A & 0 \\
0 & B
\end{array}\right) \prec_{w}\left(\begin{array}{cc}
X & 0 \\
0 & Y
\end{array}\right) \text {. }
$$

Proof. We have

$$
\sum_{j=1}^{k} \lambda_{j}(A \oplus B)=\max _{s+t=k}\left\{\sum_{j=1}^{s} \lambda_{j}(A)+\sum_{j=1}^{t} \lambda_{j}(B)\right\} .
$$

Combining this with

$$
\sum_{j=1}^{s} \lambda_{j}(A)+\sum_{j=1}^{t} \lambda_{j}(B) \leq \sum_{j=1}^{s} \lambda_{j}(X)+\sum_{j=1}^{t} \lambda_{j}(Y) \leq \sum_{j=1}^{k} \lambda_{j}(X \oplus Y)
$$

ends the proof. 
Lemma 2. Let $A, B \geq 0$. Then,

$$
\left(\begin{array}{cc}
A & 0 \\
0 & B
\end{array}\right) \prec_{w}\left(\begin{array}{cc}
A+B & 0 \\
0 & 0
\end{array}\right) .
$$

Proof. Note that

$$
\left(\begin{array}{cc}
A+B & 0 \\
0 & 0
\end{array}\right)=\left(\begin{array}{cc}
A^{1 / 2} & B^{1 / 2} \\
0 & 0
\end{array}\right)\left(\begin{array}{ll}
A^{1 / 2} & 0 \\
B^{1 / 2} & 0
\end{array}\right)
$$

so that

$$
\left(\begin{array}{cc}
A+B & 0 \\
0 & 0
\end{array}\right) \simeq\left(\begin{array}{cc}
A & A^{1 / 2} B^{1 / 2} \\
B^{1 / 2} A^{1 / 2} & B
\end{array}\right) \simeq\left(\begin{array}{cc}
A & -A^{1 / 2} B^{1 / 2} \\
-B^{1 / 2} A^{1 / 2} & B
\end{array}\right),
$$

where $\simeq$ means unitarily congruent. Combining with

$$
\left(\begin{array}{cc}
A & 0 \\
0 & B
\end{array}\right)=\frac{1}{2}\left(\begin{array}{cc}
A & A^{1 / 2} B^{1 / 2} \\
B^{1 / 2} A^{1 / 2} & B
\end{array}\right)+\frac{1}{2}\left(\begin{array}{cc}
A & -A^{1 / 2} B^{1 / 2} \\
-B^{1 / 2} A^{1 / 2} & B
\end{array}\right)
$$

gives the lemma.

Proof of Theorem 2.1. It suffices to prove the result when $A$ and $B$ are Hermitian. The general case then follows by replacing $A, B$ by

$$
\tilde{A}=\left(\begin{array}{cc}
0 & A \\
A^{*} & 0
\end{array}\right), \quad \tilde{B}=\left(\begin{array}{cc}
0 & A \\
A^{*} & 0
\end{array}\right)
$$

and by using normality of $A$ and $B$. Therefore assume that $A, B$ are Hermitian with decomposition into positive and negative parts,

$$
A=A_{+}-A_{-} \quad \text { and } \quad B=B_{+}-B_{-} .
$$

Let $g(t)=f(t)-f(0)$ and note that, for each Ky Fan $k$-norm,

$$
\|f(|A+B|)\|_{(k)}=k f(0)+\|g(|A+B|)\|_{(k)}
$$

and

$$
\|f(|A|)+f(|B|)\|_{(k)}=2 k f(0)+\|g(|A|)+g(|B|)\|_{(k)} .
$$

Hence, it suffices to prove the result for $g(t)$, or equivalently when $f(0)=0$. This assumption implies that

$$
f(|A|)=f\left(A_{+}\right)+f\left(A_{-}\right) \quad \text { and } \quad f(|B|)=f\left(B_{+}\right)+f\left(B_{-}\right) .
$$

Now, given two positive $n$-by- $n$ matrices $X$ and $Y$ with direct sum

$$
X \oplus Y=\left(\begin{array}{cc}
X & 0 \\
0 & Y
\end{array}\right),
$$

we have

$$
\lambda_{j}(|X-Y|) \leq \lambda_{j}(X \oplus Y)
$$

for all $j=1,2, \ldots, n$. Indeed, for some subspace $\mathcal{S} \subset \mathcal{H}$ we have

$$
\begin{aligned}
|X-Y| & =(X-Y)_{+}+(X-Y)_{-} \\
& =(X-Y)_{\mathcal{S}} \oplus(Y-X)_{\mathcal{S}^{\perp}} \\
& \leq X_{\mathcal{S}} \oplus Y_{\mathcal{S}^{\perp}}
\end{aligned}
$$

hence

$$
\lambda_{j}(|X-Y|) \leq \lambda_{j}\left(X_{\mathcal{S}} \oplus Y_{\mathcal{S}^{\perp}}\right) \leq \lambda_{j}(X \oplus Y)
$$


for all $j=1,2, \ldots, n$. In (4) replacing $X$ by $A_{+}+B_{+}$and $Y$ by $A_{-}+B_{-}$we then get

$$
\lambda_{j}(|A+B|) \leq \lambda_{j}\left(\left(A_{+}+B_{+}\right) \oplus\left(A_{-}+B_{-}\right)\right)
$$

for all $j=1,2, \ldots, n$. Since $f$ is non-decreasing, it follows that

$$
\lambda_{j}(f(|A+B|)) \leq \lambda_{j}\left(f\left(A_{+}+B_{+}\right)\right) \oplus\left(f\left(A_{-}+B_{-}\right)\right)
$$

for all $j=1,2, \ldots, n$, so that

$$
\|f(|A+B|)\| \leq\left\|\left(\begin{array}{cc}
f\left(A_{+}+B_{+}\right) & 0 \\
0 & f\left(A_{-}+B_{-}\right)
\end{array}\right)\right\|
$$

for all symmetric norms. By Theorem 1.1 combined with Lemma 1, followed by an application of Lemma 2, we then obtain

$$
\|f(|A+B|)\| \leq\left\|f\left(A_{+}\right)+f\left(B_{+}\right)+f\left(A_{-}\right)+f\left(B_{-}\right)\right\|,
$$

and making use of relations (3) ends the proof.

Let us now give some applications for block matrices. The most obvious one is for a Hermitian matrix

$$
\left(\begin{array}{cc}
A & B \\
B^{*} & C
\end{array}\right)
$$

partitioned into four blocks of the same size. Then by using Theorem 2.1 for the decomposition into two Hermitian matrices,

$$
\left(\begin{array}{cc}
A & B \\
B^{*} & C
\end{array}\right)=\left(\begin{array}{cc}
A & 0 \\
0 & C
\end{array}\right)+\left(\begin{array}{cc}
0 & B \\
B^{*} & 0
\end{array}\right)
$$

and then using Lemma 1, we have

$$
\left\|f\left(\left|\left(\begin{array}{cc}
A & B \\
B^{*} & C
\end{array}\right)\right|\right)\right\| \leq\left\|f(|A|)+f(|B|)+f\left(\left|B^{*}\right|\right)+f(|C|)\right\|
$$

for all concave functions $f:[0, \infty) \rightarrow[0, \infty)$ and all symmetric norms.

To obtain similar statements for more general partitions, note that the proof of Theorem 2.1 is valid for any finite family of normal operators. Thus: Let $\left\{A_{i}\right\}_{i=1}^{m}$ be normal and let $f:[0, \infty) \rightarrow[0, \infty)$ be concave. Then, for all symmetric norms,

$$
\left\|f\left(\left|A_{1}+\cdots+A_{m}\right|\right)\right\| \leq\left\|f\left(\left|A_{1}\right|\right)+\cdots+f\left(\left|A_{m}\right|\right)\right\| .
$$

We may then obtain results for some matrices partitioned into $m^{2}$ blocks of the same size.

Corollary 2.4. Let $\mathbb{A}=\left[A_{i, j}\right]$ be a block matrix with normal entries and let $f$ be a non-negative concave function on $[0, \infty)$. Then, for all symmetric norms,

$$
\|f(|\mathbb{A}|)\| \leq\left\|\sum f\left(\left|A_{i, j}\right|\right)\right\| .
$$

Proof. We prove this corollary via Theorem 2.1 for a partition into four blocks

$$
\mathbb{A}=\left(\begin{array}{ll}
S & R \\
T & Q
\end{array}\right) .
$$

The proof for a partition into $m^{2}$ blocks is similar by using the version of Theorem 2.1 for $m$ operators. Let

$$
\tilde{\mathbb{A}}=\left(\begin{array}{cc}
0 & \mathbb{A} \\
\mathbb{A}^{*} & 0
\end{array}\right)
$$


and note that

so that

$$
|\tilde{\mathbb{A}}|=\left(\begin{array}{cc}
\left|\mathbb{A}^{*}\right| & 0 \\
0 & |\mathbb{A}|
\end{array}\right)
$$

$$
|\tilde{\mathbb{A}}| \simeq\left(\begin{array}{cc}
|\mathbb{A}| & 0 \\
0 & |\mathbb{A}|
\end{array}\right)
$$

where the symbol $\simeq$ stands for unitarily equivalent. On the other hand,

$$
\tilde{\mathbb{A}}=\tilde{\mathbb{S}}+\tilde{\mathbb{T}}
$$

where

$$
\tilde{\mathbb{S}}=\left(\begin{array}{cccc}
0 & 0 & S & 0 \\
0 & 0 & 0 & Q \\
S^{*} & 0 & 0 & 0 \\
0 & Q^{*} & 0 & 0
\end{array}\right), \quad \tilde{\mathbb{T}}=\left(\begin{array}{cccc}
0 & 0 & 0 & R \\
0 & 0 & T & 0 \\
0 & T^{*} & 0 & 0 \\
R^{*} & 0 & 0 & 0
\end{array}\right)
$$

are Hermitian. Therefore, Theorem 2.1 yields

$$
\|f(|\tilde{\mathbb{A}}|)\| \leq\|f(|\tilde{\mathbb{S}}|)+f(|\tilde{\mathbb{T}}|)\|
$$

for all symmetric norms; that is, using the shorthand symbol $\prec_{w}$,

$$
f(|\tilde{\mathbb{A}}|) \prec_{w}\left(\begin{array}{cccc}
f\left(\left|S^{*}\right|\right)+f\left(\left|R^{*}\right|\right) & 0 & 0 & 0 \\
0 & f\left(\left|T^{*}\right|\right)+f\left(\left|Q^{*}\right|\right) & 0 & 0 \\
0 & 0 & f(|S|)+f(|T|) & 0 \\
0 & 0 & 0 & f(|R|)+f(|Q|)
\end{array}\right) .
$$

Gathering the first two lines and the last two lines, we have via Lemmas 2 and 1,

$$
f(|\tilde{\mathbb{A}}|) \prec{ }_{w}\left(\begin{array}{cc}
f\left(\left|S^{*}\right|\right)+f\left(\left|T^{*}\right|\right)+f\left(\left|R^{*}\right|\right)+f\left(\left|Q^{*}\right|\right) & 0 \\
0 & f(|S|)+f(|T|)+f(|R|)+f(|Q|)
\end{array}\right) .
$$

By using (6) we then obtain, using normality of $S, T, R, Q$,

$$
f(|\mathbb{A}|) \prec_{w} f(|S|)+f(|T|)+f(|R|)+f(|Q|),
$$

which is equivalent to inequalities for symmetric norms.

Let us point out a variation of Corollary 2.4 in which some operators are not necessarily normal.

Corollary 2.5. Let $\mathbb{T}$ be a triangular block matrix,

$$
\mathbb{T}=\left(\begin{array}{cc}
A & N \\
0 & B
\end{array}\right)
$$

in which $N$ is normal. Let $f:[0, \infty) \rightarrow[0, \infty)$ be concave. Then, for all symmetric norms,

$$
\|f(|\mathbb{T}|)\| \leq\left\|f\left(\left|A^{*}\right|\right)+f(|N|)+f(|B|)\right\| .
$$

Proof. Consider the polar decompositions $A=\left|A^{*}\right| U$ and $B=V|B|$, note that

$$
\left|\left(\begin{array}{cc}
A & N \\
0 & B
\end{array}\right)\right| \simeq\left|\left(\begin{array}{cc}
I & 0 \\
0 & V^{*}
\end{array}\right)\left(\begin{array}{cc}
A & N \\
0 & B
\end{array}\right)\left(\begin{array}{cc}
U^{*} & 0 \\
0 & I
\end{array}\right)\right|=\left|\left(\begin{array}{cc}
\left|A^{*}\right| & N \\
0 & |B|
\end{array}\right)\right|
$$

and apply Theorem 2.1.

The assumption in Corollary 2.4 requiring normality of each block is rather special. The next corollary generalizes (5) and meets the simple requirement that the full matrix is Hermitian. 
Corollary 2.6. Let $\mathbb{A}=\left[A_{i, j}\right]$ be a Hermitian matrix partitioned into blocks of the same size and let $f:[0, \infty) \rightarrow[0, \infty)$ be concave. Then, for all symmetric norms,

$$
\|f(|\mathbb{A}|)\| \leq\left\|\sum f\left(\left|A_{i, j}\right|\right)\right\| \text {. }
$$

Proof. The proof of Corollary 2.4 actually shows that for a general block matrix $\mathbb{A}=\left(A_{i, j}\right)$ partitioned into blocks of the same size, we have

$$
\left(\begin{array}{cc}
f(|\mathbb{A}|) & 0 \\
0 & f(|\mathbb{A}|)
\end{array}\right) \prec_{w}\left(\begin{array}{cc}
\sum f\left(\left|A_{i, j}^{*}\right|\right) & 0 \\
0 & \sum f\left(\left|A_{i, j}\right|\right)
\end{array}\right)
$$

for all non-negative concave functions $f$. Assuming $\mathbb{A}$ Hermitian, we have $A_{i, j}^{*}=$ $A_{j, i}$ and Corollary 2.6 follows.

\section{RELATED RESUlTS FOR EXPANSIVE CONGRUENCES}

Let $A$ be normal and let $Z$ be expansive, i.e., $Z^{*} Z \geq I$. The following extension of Theorem 1.3 holds.

Theorem 3.1. Let $A$ be normal and let $Z$ be expansive. If $f:[0, \infty) \rightarrow[0, \infty)$ is concave, then, for all symmetric norms,

$$
\left\|f\left(\left|Z^{*} A Z\right|\right)\right\| \leq\left\|Z^{*} f(|A|) Z\right\| .
$$

Indeed, we can derive Theorem 3.1 from Theorem 1.3 in a quite similar way to that in which we derive Theorem 2.1 from Theorem 1.1. The proof of Theorem 3.1 starts by noticing that we can assume that $A$ is Hermitian. Then, using the decomposition into positive and negative parts,

$$
A=A_{+}-A_{-},
$$

we have, as in the proof of Theorem 2.1,

$$
\lambda_{j}\left(\left|Z^{*} A Z\right|\right) \leq \lambda_{j}\left(\left|Z^{*} A_{+} Z\right| \oplus\left|Z^{*} A_{-} Z\right|\right)
$$

and we may proceed as previously.

When we deal with the trace norm, the fact that $f$ is positive on the whole half-line is not essential, as in the Rotfel'd inequality. Hence we have the following corollary, extending to normal operators a result from [4.

Corollary 3.2. Let $A$ be normal and let $Z$ be expansive. If $f(t)$ is a concave function on the positive half-line with $f(0) \geq 0$, then

$$
\operatorname{Tr} f\left(\left|Z^{*} A Z\right|\right) \leq \operatorname{Tr} Z^{*} f(|A|) Z .
$$

Corollary 3.2 follows from Theorem 3.1 by approaching $f(t)$ with $g(t)+a t$ for some scalar $a$ and some non-negative concave function $g(t)$. Theorems 2.1 and 3.1 can be combined in a unique statement, extending the main result in [7:

Theorem 3.3. Let $\left\{A_{i}\right\}_{i=1}^{m}$ be normal, let $\left\{Z_{i}\right\}_{i=1}^{m}$ be expansive and let $f$ be a non-negative concave function on $[0, \infty)$. Then, for all symmetric norms,

$$
\left\|f\left(\left|\sum Z_{i}^{*} A_{i} Z_{i}\right|\right)\right\| \leq\left\|\sum Z_{i}^{*} f\left(\left|A_{i}\right|\right) Z_{i}\right\| .
$$


It would be elegant and interesting to state this theorem in the more general framework of positive linear maps $\Phi$ between matrix algebras. This leads to the problem of characterizing the positive linear maps $\Phi$ such that

$$
\|f(|\Phi(N)|)\| \leq\|\Phi(f(|N|))\|
$$

for all normal operators $N$, all non-negative concave functions and all symmetric norms. Some further questions are considered in [6]. For the sake of completeness, we mention that when $f(t)$ is a non-negative convex function vanishing at 0 , then the inequalities of Theorems 1.1-1.3 are reversed. For instance we have [7]

Theorem 3.4. Let $\left\{A_{i}\right\}_{i=1}^{m}$ be positive and let $\left\{Z_{i}\right\}_{i=1}^{m}$ be expansive. Then, for all symmetric norms and all $p>1$,

$$
\left\|\sum Z_{i}^{*} A_{i}^{p} Z_{i}\right\| \leq\left\|\left(\sum Z_{i}^{*} A_{i} Z_{i}\right)^{p}\right\|
$$

If $Z_{i}=I$ for all $i$, it is a famous result of Ando-Zhan [1] and of BhatiaKittaneh [3] in the case of integer exponents. The very special case $\operatorname{Tr}\left(A_{1}^{p}+A_{2}^{p}\right) \leq$ $\operatorname{Tr}\left(A_{1}+A_{2}\right)^{p}$ is McCarthy's inequality [13, p. 20]. Note that the positivity assumption in Theorem 3.4 cannot be replaced by a normality one.

When we consider contractive congruences and positive operators, there exist several Jensen-type inequalities, not only for norms but also for eigenvalues (cf. [4], 5]). The proofs are much simpler than in the expansive case, where some unexpected counterexamples may occur (see discussion and counterexamples in [4], [5]). We give an example of such results:

Let $\left\{A_{i}\right\}_{i=1}^{m}$ be positive and $\left\{Z_{i}\right\}_{i=1}^{m}$ such that $\sum Z_{i}^{*} Z_{i} \leq$ I. If $f$ is a monotone concave function on $[0, \infty), f(0) \geq 0$, then

$$
f\left(\sum Z_{i}^{*} A_{i} Z_{i}\right) \geq V\left(\sum Z_{i}^{*} f\left(A_{i}\right) Z_{i}\right) V^{*}
$$

for some unitary $V$.

\section{REFERENCES}

[1] T. Ando and X. Zhan, Norm inequalities related to operator monotone functions, Math. Ann. 315 (1999) 771-780. MR.1727183 (2000m:47008)

[2] R. Bhatia, Matrix Analysis, Grad. Texts in Math., 169, Springer-Verlag, New York, 1997. MR 1477662 (98i:15003)

[3] R. Bhatia and F. Kittaneh, Norm inequalities for partitioned operators and an application, Math. Ann. 287 (1990) 719-726. MR 1066826 (91f:47010)

[4] J.-C. Bourin, Convexity or concavity inequalities for Hermitian operators, Math. Ineq. Appl. 7 (4) (2004) 607-620. MR.2097515 (2005h:47034)

[5] J.-C. Bourin, A concavity inequality for symmetric norms, Linear Alg. Appl. 413 (2006) 212-217. MR2202104 (2007d:47023)

[6] J.-C. Bourin, Matrix subadditivity inequalities and block-matrices, Internat. J. Math. 20, no. 6 (2009), 679-691.

[7] J.-C. Bourin and E.-Y. Lee, Concave functions of positive operators, sums and congruences, to appear in J. Operator Theory.

[8] J.-C. Bourin and M. Uchiyama, A matrix subadditivity inequality for $f(A+B)$ and $f(A)+$ $f(B)$, Linear Alg. Appl. 423 (2007) 512-518. MR2312422 (2007m:15023)

[9] C. King, M. Nathanson, New trace norm inequalities for $2 \times 2$ blocks of diagonal matrices, Linear Alg. Appl. 389 (2004) 77-93. MR2080396 (2005h:15058)

[10] T. Kosem, Inequalities between $\|f(A+B)\|$ and $\|f(A)+f(B)\|$, Linear Alg. Appl. 418 (2006) 153-160. MR2257586 (2007g:47026)

[11] S. Ju. Rotfel'd, The singular values of the sum of completely continuous operators, Problems of Mathematical Physics, Vol. 3, Consultants Bureau, 1969, 73-78. MR0339968 
[12] B. Simon, Trace ideals and their applications, Cambridge Univ. Press, Cambridge-New York, 1979. MR541149 (80k:47048)

[13] O. Tychonov, Subadditivity inequalities in von Neumann algebras and characterization of tracial functionals, Positivity 9 (2005) 259-264. MR.2189748 (2006f:46056)

Laboratoire de Mathématiques, Université de Franche-Comté, 25030 Besançon, FRANCE

E-mail address: jcbourin@univ-fcomte.fr 